LETTER TO THE EDITOR

\title{
Entanglement and tensor product decomposition for two fermions
}

\author{
P Caban, K Podlaski, J Rembieliński, K A Smoliński and \\ Z Walczak \\ Department of Theoretical Physics, University of Lodz, Pomorska 149/153, \\ 90-236 Łódź, Poland
}

\begin{abstract}
The problem of the choice of tensor product decomposition in a system of two fermions with the help of Bogoliubov transformations of creation and annihilation operators is discussed. The set of physical states of the composite system is restricted by the superselection rule forbidding the superposition of fermions and bosons. It is shown that the Wootters concurrence is not the proper entanglement measure in this case. The explicit formula for the entanglement of formation is found. This formula shows that the entanglement of a given state depends on the tensor product decomposition of a Hilbert space. It is shown that the set of separable states is narrower than in the two-qubit case. Moreover, there exist states which are separable with respect to all tensor product decompositions of the Hilbert space.

PACS numbers: 03.67.Mn, 03.65.Ud
\end{abstract}

Entanglement is the key notion of quantum information theory and plays a significant role in most of its applications. The entanglement of a physical system is always relative to a particular set of experimental capabilities (see, e.g. 1] 2]), which is connected with decompositions of the system into subsystems. From the theoretical point of view this is closely related to possible choices of the tensor product decomposition (TPD) of the Hilbert space of the system. As a consequence the following question arises: How much entangled is a given state with respect to a particular TPD?

In the present paper we discuss the problem of the choices of TPD in a system of two fermions, neglecting their spatial degrees of freedom and modifying tensor product in the rings of operators because of anticommuting canonical variables. We show that TPDs are connected with each other by Bogoliubov transformations of creation and annihilation operators. We also study the behavior of the entanglement of the system under these transformations. An importance of such investigation can be illustrated for example by the fact that the Bogoliubov transformations used in derivation of the Unruh effect also lead to the change of entanglement [3]. Different approach to the entanglement in the system of two identical fermions, based on the asymmetric decomposition of the algebra generated by $a_{i}, a_{i}^{\dagger}(i=1,2)$ into tensor product of two subalgebras was taken up in [4. Some aspects of the entanglement for two-fermion system were also discussed in [5]. 
The theory of entanglement can be seen as the general theory of states transformations that can be performed on multipartite systems, with restriction that only local operations and classical communications (LOCC) can be implemented [6]. For the same reason, it was expected that additional restrictions should lead to new interesting physical effects and applications. Recently, it has been shown that such a restriction can be given by a superselection rule (SSR) [7, 8].

In this work we restrict the set of physical states of the composite system by the requirement that we prohibit superpositions of fermions and bosons. This leads us to the SSR that is a weaker restriction (i.e., it admits larger set of states) than SSR based on the conservation of the number of particles [7. Moreover, we find the entanglement of formation taking into account the restriction imposed by our SSR.

Let us consider the Hilbert space $\mathcal{H} \cong \mathbb{C}^{4}$ with an orthonormal basis $\{|m, n\rangle\}_{m, n=0,1}$. With this basis we associate the following two operators:

$$
\begin{aligned}
& a_{1}=|0,0\rangle\langle 1,0|-| 0,1\rangle\langle 1,1|, \\
& a_{2}=|0,0\rangle\langle 0,1|+| 1,0\rangle\langle 1,1| .
\end{aligned}
$$

One can easily check that these operators and their Hermitian conjugations fulfill the following relations:

$$
\left\{a_{i}, a_{j}\right\}=0, \quad\left\{a_{i}, a_{j}^{\dagger}\right\}=\delta_{i j}, \quad i, j=1,2,
$$

where $\{.,$.$\} stands for anticommutator. Operators a_{i}^{\dagger}$ generate all the basis vectors from the "vacuum state" $|0,0\rangle$ via the relations

$$
\begin{aligned}
& |1,0\rangle=a_{1}^{\dagger}|0,0\rangle, \\
& |0,1\rangle=a_{2}^{\dagger}|0,0\rangle, \\
& |1,1\rangle=a_{2}^{\dagger} a_{1}^{\dagger}|0,0\rangle,
\end{aligned}
$$

while the vacuum is annihilated by $a_{i}$, i.e. $a_{i}|0,0\rangle=0, i=1,2$. We use the occupation number basis, i.e., the Fock basis, not the so called "computational basis". Thus with every orthonormal basis we can associate some representation of the algebra (2). On the other hand it is clear that Eqs. (2) can be interpreted as canonical anticommutation relations for two-fermion system.

Every two orthonormal bases in $\mathcal{H}$ are connected by some unitary transformation belonging to the group $U(4)$. In the ring of operators these changes of bases are related to Bogoliubov transformations of creation and annihilation operators which will be discussed later on.

One can naively expect that, as in the bosonic case, the operators $a_{1}$ and $a_{2}$ should have the form $a \otimes$ id and id $\otimes a$, respectively, where $a$ is an annihilation operator for single fermion acting in $\mathbb{C}^{2}$, that is

$$
\begin{aligned}
& a|0\rangle=0, \quad a|1\rangle=|0\rangle, \\
& a^{\dagger}|0\rangle=|1\rangle, \quad a^{\dagger}|1\rangle=0, \\
& \left\{a, a^{\dagger}\right\}=\mathrm{id},
\end{aligned}
$$

and id denotes the identity operator. However it is not the case because $a \otimes$ id and id $\otimes a$ necessarily commute so they cannot fulfill the canonical anticommutation relations (2). In order to construct $a_{1}, a_{2}$ out of the single annihilation operator $a$ and to provide natural tensor product interpretation of basis vectors as $|m, n\rangle=|m\rangle \otimes|n\rangle$ we have to modify only the tensor product of the operators acting in $\mathbb{C}^{2}$. Hereafter 
we will denote the new tensor multiplication by the usual symbol $\otimes$. Such a modified tensor product is defined by the graded (supersymmetric) multiplication rule

$$
(A \otimes \mathfrak{b})(\mathfrak{a} \otimes B)=(-1)^{F(\mathfrak{a}) F(\mathfrak{b})} A \mathfrak{a} \otimes \mathfrak{b} B,
$$

where $\mathfrak{a}, \mathfrak{b}$ are monomials in $a, a^{\dagger}$, i.e. $\mathfrak{a}, \mathfrak{b} \in\left\{\operatorname{id}, a, a^{\dagger}, a a^{\dagger}, a^{\dagger} a\right\}, A, B$ are arbitrary operators acting in $\mathbb{C}^{2}$ and the "fermion number" $F(\mathfrak{a})$ is equal to the number of creation operators minus the number of annihilation operators building the monomial a, i.e. $F($ id $)=0, F\left(a^{\dagger}\right)=-F(a)=1, F\left(a a^{\dagger}\right)=F\left(a^{\dagger} a\right)=0$. Consequently the Hermitian conjugation in this tensor product is of the form

$$
(\mathfrak{a} \otimes \mathfrak{b})^{\dagger}=(-1)^{F(\mathfrak{a}) F(\mathfrak{b})}\left(\mathfrak{a}^{\dagger} \otimes \mathfrak{b}^{\dagger}\right) .
$$

The tensor multiplication introduced above is a special case of a more general structure known in mathematical physics as the braided tensor product [9]. As we can see from the relation (5), the new braided tensor product for monomials even in $a, a^{\dagger}$ behaves like the standard tensor product.

Finally, the relationship between the tensor product of operators and the tensor product of vectors is given by

$$
\begin{aligned}
& (\mathrm{id} \otimes \mathrm{id})(|m\rangle \otimes|n\rangle)=|m\rangle \otimes|n\rangle, \\
& (a \otimes \mathrm{id})(|m\rangle \otimes|n\rangle)=(-1)^{n} a|m\rangle \otimes|n\rangle, \\
& \left(a^{\dagger} \otimes \mathrm{id}\right)(|m\rangle \otimes|n\rangle)=(-1)^{n} a^{\dagger}|m\rangle \otimes|n\rangle, \\
& (\mathrm{id} \otimes a)(|m\rangle \otimes|n\rangle)=|m\rangle \otimes a|n\rangle, \\
& \left(\mathrm{id} \otimes a^{\dagger}\right)(|m\rangle \otimes|n\rangle)=|m\rangle \otimes a^{\dagger}|n\rangle .
\end{aligned}
$$

Now the annihilation and creation operators acting in the space $\mathbb{C}^{2} \otimes \mathbb{C}^{2}$ and satisfying (21) take the desired form

$$
\begin{aligned}
& a_{1}=a \otimes \mathrm{id}, \quad a_{2}=\mathrm{id} \otimes a, \\
& a_{1}^{\dagger}=a^{\dagger} \otimes \mathrm{id}, \quad a_{2}^{\dagger}=\mathrm{id} \otimes a^{\dagger} .
\end{aligned}
$$

Notice, that in the above equations $\otimes$ denotes the new tensor multiplication thus Eqs. (4a)-(17e) imply that operators (8a)-(8b) fulfill the canonical anticommutation relations (2). In particular, the matrix elements of operators (8a)- (8b) and (1a)-(1b) are identical in the basis $\{|m, n\rangle\}_{m, n=0,1}$.

Similarly, like in the case of quantum theory of fermionic fields in the system under consideration observables are restricted to combinations of even products of creation and annihilation operators. In particular the local observables are combinations of id $\otimes$ id and $N_{1}=a^{\dagger} a \otimes$ id or id $\otimes$ id and $N_{2}=$ id $\otimes a^{\dagger} a$. It is implied by the SSR related to the requirement that the operator $(-1)^{\hat{F}}$, where $\hat{F}$ is the fermion number operator, should commute with all observables [10]. It means that superpositions of bosons and fermions are forbidden. In the basis (Ba)- $(-1)^{\hat{F}}=\operatorname{diag}\{1,-1,-1,1\}$. Alternatively, this SSR is a consequence of the requirement that the squared time reflection operator must commute with all observables (see e.g. [11). Indeed, antiunitary time inversion operator is defined here as follows

$$
\begin{aligned}
& \mathrm{T} a_{1} \mathrm{~T}^{-1}=a_{2}, \quad \mathrm{~T} a_{2} \mathrm{~T}^{-1}=-a_{1}, \\
& \mathrm{~T}|0,0\rangle=|0,0\rangle .
\end{aligned}
$$


Thus $\mathrm{T}^{2}=(-1)^{\hat{F}}$. Due to the SSR the density matrix has to commute with $(-1)^{\hat{F}}$, so the general state of this system is represented by the following density matrix

$$
\rho=\left(\begin{array}{cccc}
w_{1} & 0 & 0 & b_{1} \\
0 & w_{2} & b_{2} & 0 \\
0 & b_{2}^{*} & v_{2} & 0 \\
b_{1}^{*} & 0 & 0 & v_{1}
\end{array}\right),
$$

where $w_{i}, v_{i} \geq 0, \sum_{i=1}^{2}\left(w_{i}+v_{i}\right)=1$ and $\left|b_{i}\right|^{2} \leq w_{i} v_{i}, i=1,2$. Consequently, possible states of subsystems obtained from (11) by partial traces are

$$
\begin{aligned}
& \rho_{1}=\left(\begin{array}{cc}
w_{1}+v_{2} & 0 \\
0 & w_{2}+v_{1}
\end{array}\right), \\
& \rho_{2}=\left(\begin{array}{cc}
w_{1}+w_{2} & 0 \\
0 & v_{1}+v_{2}
\end{array}\right) .
\end{aligned}
$$

Note that the diagonal form of $(12 a)-12 b)$ is in conformance with the SSR in spaces of subsystems. Moreover, the states (12a) - 12b exhaust all possible states of the subsystems. Therefore our subsystems are independent in the sense of the definition of the algebraic independence of subsystems 4, 12. This independence is due to the SSR (compare 4] where it was shown that in general algebras of observables of two identical fermions are nonindependent). The natural question arises: What is the form of the separable states for this system? According to Werner's definition [13] the state is separable if it can be written in the form $\rho=\sum_{i} p_{i} \rho_{1}^{i} \otimes \rho_{2}^{i}$, where $\rho_{1}^{i}$ and $\rho_{2}^{i}$ are admissible states of subsystems and $\sum_{i} p_{i}=1, p_{i} \geq 0$. Therefore, taking into account that $\rho_{1}^{i}$ and $\rho_{2}^{i}$ are of the form (12a) - 12b), the separable states have the surprisingly simple diagonal form

$$
\rho_{\text {sep }}=\left(\begin{array}{cccc}
\lambda_{1} & 0 & 0 & 0 \\
0 & \lambda_{2} & 0 & 0 \\
0 & 0 & \lambda_{3} & 0 \\
0 & 0 & 0 & \lambda_{4}
\end{array}\right),
$$

with $\sum_{i} \lambda_{i}=1, \lambda_{i} \geq 0$. Consequently, nondiagonal density matrices are nonseparable. Thus in this case the standard method of calculating entanglement measures should be taken with care. Indeed, as an example let us consider the Werner state [13, 14]

$$
\rho_{W}=\left(\begin{array}{cccc}
\frac{1+\gamma}{4} & 0 & 0 & \frac{\gamma}{2} \\
0 & \frac{1-\gamma}{4} & 0 & 0 \\
0 & 0 & \frac{1-\gamma}{4} & 0 \\
\frac{\gamma}{2} & 0 & 0 & \frac{1+\gamma}{4}
\end{array}\right), \quad \gamma \in[-1 / 3,1]
$$

which belongs to the admissible states (11). The Wootters concurrence [15] of this state is equal to zero for $\gamma \in[-1 / 3,1 / 3]$, therefore for two qubits the Werner state is separable for such values of $\gamma$. On the other hand, in our case this state is separable only when $\gamma=0$. Thus the Wootters concurrence does not define entanglement measure in our case.

Instead, let us calculate directly the entanglement of formation [6], i.e.:

$$
E(\rho)=\min \sum_{i} p_{i} S\left(\rho_{A}^{i}\right),
$$

where $S\left(\rho_{A}\right)=-\operatorname{Tr} \rho_{A} \log _{2} \rho_{A}$ is the von Neumann entropy and the minimum is taken over all the possible realizations of the state $\rho=\sum_{i} p_{i}\left|\psi_{i}\right\rangle\left\langle\psi_{i}\right|$ with 
$\rho_{A}^{i}=\operatorname{Tr}_{B}\left(\left|\psi_{i}\right\rangle\left\langle\psi_{i}\right|\right)$. Taking into account the special form of the density matrix (111) we can find the explicit formula for the entanglement of formation

$$
E(\rho)=\sum_{i=1}^{2}\left(w_{i}+v_{i}\right) S_{i}
$$

where

$S_{i}= \begin{cases}0 & \text { if } w_{i}=v_{i} \text { and } b_{i}=0 \\ -\frac{1}{2}\left[\left(1-\xi_{i}\right) \log _{2} \frac{1-\xi_{i}}{2}+\left(1+\xi_{i}\right) \log _{2} \frac{1+\xi_{i}}{2}\right] & \text { otherwise }\end{cases}$

and

$$
\xi_{i}=\frac{w_{i}-v_{i}}{\sqrt{\left(w_{i}-v_{i}\right)^{2}+4\left|b_{i}\right|^{2}}} .
$$

It is interesting that the formula similar to (17) was obtained in [16] for the so called correlational entropy of the two-level system. Note that maximal value of $E(\rho)$ is equal to 1 . In the case of the Werner state (14) the entanglement of formation (16) is

$$
E\left(\rho_{W}\right)= \begin{cases}\frac{1+\gamma}{2} & \text { if } \gamma \neq 0, \\ 0 & \text { if } \gamma=0 .\end{cases}
$$

Thus, as expected, $E\left(\rho_{W}\right) \neq 0$ for entangled (nondiagonal) states and $E\left(\rho_{W}\right)=0$ for a separable (diagonal) state. For $\gamma=1$ we have the maximally entangled Werner state. Notice, that the restriction of admissible states by SSR implies that in our case we have no asymmetry in the definition of the entanglement of formation, in contrast to observations of 4 .

Let us consider the problem of decomposition of our system into two subsystems. Such a decomposition corresponds to the different choices of canonical variables $a_{i}, a_{i}^{\dagger}$. This is extremely important because each choice of $a_{i}, a_{i}^{\dagger}$ defines in the Hilbert space $\mathcal{H}$ the corresponding tensor product structure (5) - (7e) such that the creation and annihilation operators take the form analogous to (8ab) $-8 b$. Each TPD defines a set of local observables of the form $A \otimes$ id and id $\otimes B$ [cf. the discussion after $[8 a b-(8 b)]$. Moreover, the notion of a local observer is determined by his experimental access to local observables (see e.g. [1]).

Different choices of canonical variables $a_{i}, a_{i}^{\dagger}$ are connected by transformations which preserve the canonical anticommutation relations (2) (Bogoliubov transformations $\dagger$ ). Therefore Bogoliubov transformations give us all possible decompositions of the two-fermion system into two subsystems (two fermions). Such decompositions of the system correspond to the tensor product decompositions of the space $\mathcal{H} \cong \mathbb{C}^{2} \otimes \mathbb{C}^{2}$, appropriate to the definition of the subsystems. In the case under consideration the problem of finding all possible TPDs consistent with our SSR is equivalent to determining all possible Bogoliubov transformations commuting with the superselection operator $\mathrm{T}^{2}=(-1)^{\hat{F}}$.

Let us notice first that operators $a_{i}, a_{i}^{\dagger}$ in every orthonormal basis can be represented in the form (Ba)-(BC) and vice versa such operators define orthonormal

$\dagger$ By Bogoliubov transformations we mean here all transformations of creation and annihilation operators (linear as well as nonlinear) which do not change the canonical anticommutation relations. 
basis via (3a)-3c). Thus different choices of these operators are connected with different choices of orthonormal bases in the Hilbert space. Therefore

$$
a_{i}^{\prime}=U a_{i} U^{\dagger},
$$

where $U$ is an unitary matrix. As we have mentioned above, the consistency with SSR means that $U$ commutes with $T^{2}=(-1)^{\hat{F}}=\operatorname{diag}\{1,-1,-1,1\}$. So $U$ can be represented as the following product of unitary matrices

$U=\left(\begin{array}{cccc}1 & 0 & 0 & 0 \\ 0 & \alpha^{*} & -\beta & 0 \\ 0 & \beta^{*} & \alpha & 0 \\ 0 & 0 & 0 & 1\end{array}\right)\left(\begin{array}{cccc}\zeta & 0 & 0 & -\omega^{*} \\ 0 & 1 & 0 & 0 \\ 0 & 0 & 1 & 0 \\ \omega & 0 & 0 & \zeta^{*}\end{array}\right)\left(\begin{array}{cccc}1 & 0 & 0 & 0 \\ 0 & 1 & 0 & 0 \\ 0 & 0 & 1 & 0 \\ 0 & 0 & 0 & e^{-i \chi}\end{array}\right)$,

with $|\alpha|^{2}+|\beta|^{2}=1$ and $|\zeta|^{2}+|\omega|^{2}=1$, where we took into account the fact that Eq. (20) determines $U$ up to an overall phase. Thus all Bogoliubov transformations admissible by the SSR form the group $S U(2) \otimes U(2)$. Applying these transformations to the explicit matrix form of $a_{i}, a_{i}^{\dagger}$ calculated from (1a)-(1b), one can show that in the ring of creation and annihilation operators the transformations (20) are realized as

- $S U(2)$ transformations which do not mix creation and annihilation operators

$$
\left(\begin{array}{l}
a_{1}^{\prime} \\
a_{2}^{\prime}
\end{array}\right)=\left(\begin{array}{cc}
\alpha & \beta \\
-\beta^{*} & \alpha^{*}
\end{array}\right)\left(\begin{array}{l}
a_{1} \\
a_{2}
\end{array}\right)
$$

- $S U(2)$ transformations which mix creation and annihilation operators

$$
\left(\begin{array}{c}
a_{1}^{\prime} \\
a_{2}^{\prime \dagger}
\end{array}\right)=\left(\begin{array}{cc}
\zeta & \omega \\
-\omega^{*} & \zeta^{*}
\end{array}\right)\left(\begin{array}{l}
a_{1} \\
a_{2}^{\dagger}
\end{array}\right)
$$

- nonlinear one-parameter transformations

$$
\begin{aligned}
& a_{1}^{\prime}=a_{1} e^{i \chi N_{2}}=a_{1}\left[1+\left(e^{i \chi}-1\right) N_{2}\right], \\
& a_{2}^{\prime}=a_{2} e^{i \chi N_{1}}=a_{2}\left[1+\left(e^{i \chi}-1\right) N_{1}\right] .
\end{aligned}
$$

Note that 24a - 24b for $\chi=\pi$ are the so called Klein-Wigner transformations (cf. (4)). The Bogoliubov transformations which lead to physically distinguishable TPDs should change the local observables $N_{i}=a_{i}^{\dagger} a_{i}$. Therefore such transformations have the form: (22) with both $\alpha \neq 0, \beta \neq 0$ and/or (23) with both $\zeta \neq 0, \omega \neq 0$.

Now, the natural question arises: How does the same state look like for local observers connected with different TPDs? The answer is quite obvious: If their TPDs are connected by Bogoliubov transformations then density matrices representing the state are connected by similarity transformations, i.e. $\rho^{\prime}=U \rho U^{\dagger}$. However, in general, such transformation change the entanglement measure $E(\rho)$, i.e. entanglement depends on the choice of TPD (and hence the local observers). In particular, for any state, there exists a pair of observers for whom this state is separable, since the density matrix (11) can always be diagonalized by means of the transformations (21). We point out that there exists a class of superseparable states $\rho_{\mathrm{ss}}=\frac{1}{2} \operatorname{diag}\{s, 1-s, 1-s, s\}, s \in[0,1]$, which are separable for every observers. Note also that in the case of two qubits only one superseparable state exists, namely the maximally mixed state $\rho_{0}=\frac{1}{4} I$.

Now we show that it is possible to construct dynamics consistent with our SSR. For such a dynamics admissible TPDs are related to symmetries of the Hamiltonian. An example of that dynamics is the Thirring model [17] in $1+0$ dimensional space-time 
describing a fermionic quantum mechanical system. The corresponding Lagrangian is of the form:

$$
L=\sum_{i=1}^{2}\left(i \psi_{i}^{\dagger} \partial_{t} \psi_{i}-m \psi_{i}^{\dagger} \psi_{i}\right)-\lambda\left(\sum_{i=1}^{2} \psi_{i}^{\dagger} \psi_{i}\right)^{2} .
$$

The solutions of the equations of motion derived from the Lagrangian (25) are

$$
\psi_{i}(t)=a_{i} e^{-i t\left(m+\lambda+2 \lambda N_{j}\right)}, \quad i \neq j,
$$

where the time-independent operators $a_{i}$ and $a_{i}^{\dagger}$ satisfying (2) can be represented in the form 8 - $8 b$. The Hamiltonian of this system is

$$
H=(m+\lambda)\left(N_{1}+N_{2}\right)+2 \lambda N_{1} N_{2}
$$

and describes two fermionic oscillators with the quartic interaction term. Notice that $\mathrm{T}^{2}=(-1)^{\hat{F}}$ commutes with $H$, thus Thirring model dynamics undergoes our SSR.

In the special case of $\lambda=-\frac{1}{2} m$ all the Bogoliubov transformations (22)-(24b) form the symmetry group of $H$, i.e. $H\left(a, a^{\dagger}\right)=H\left(a^{\prime}, a^{\prime \dagger}\right)$. Thus, this symmetry group gives us a freedom with a choice of a concrete decomposition of the system into two subsystems. Consequently the related TPDs are connected by the Bogoliubov transformations (22)- (24b).

In conclusion, we have investigated the dependence of entanglement for twofermion system on tensor product decompositions in the presence of the superselection rule. We have shown that the Wootters concurrence is not a proper entanglement measure in this case. The crucial point in finding an explicit form of entanglement of formation for such a system was determining the states of subsystems, admissible by the superselection rule. We would like to stress that these states are not qubit states. It is interesting that the set of separable states is narrower than in two-qubit case, namely it consists of only the states represented by diagonal density matrices. Moreover, we found the class of superseparable states, i.e. the states which are separable with respect to all tensor product decompositions of Hilbert space.

\section{Acknowledgments}

We would like to thank P Horodecki and T Brzeziński for helpful discussions. This paper has been partially supported by the Polish Ministry of Scientific Research and Information Technology under the grant No PBZ-MIN-008/P03/2003.

\section{References}

[1] Zanardi P, Lidar D A and Lloyd S 2004 Phys. Rev. Lett. 92060402

[2] Zanardi P 2001 Phys. Rev. Lett. 87077901

[3] Vedral V 2003 Central Eur. J. Phys. 1289

[4] Moriya H 2002 Lett. Math. Phys. 60109

[5] Shi Y 2003 Phys. Rev. A 67024301

Ghirardi G C and Marinatto L 2004 Phys. Rev. A 70012109

Schliemann J, Cirac J I, Kuś M, Lewenstein M and Loss D 2001 Phys. Rev. A 64022303

[6] Bennett C H, DiVincenzo D P, Smolin J A and Wootters W K 1996 Phys. Rev. A 543824

[7] Verstraete F and Cirac J I 2003 Phys. Rev. Lett. 91010404

Bartlett S D and Wiseman H M 2003 Phys. Rev. Lett. 91097903

[8] Terhal B M, DiVincenzo D P and Leung D W 2001 Phys. Rev. Lett. 865807

Rudolph T and Sanders B C 2001 Phys. Rev. Lett. 87077903

Wiseman H M and Vaccaro J A 2003 Phys. Rev. Lett. 91097902

Schuch N, Verstraete F and Cirac J I 2004 Phys. Rev. Lett. 92087904 
[9] Majid S 1991 J. Math. Phys. 323246 Majid S 2000 Foundations of Quantum Group Theory (Cambridge: Cambridge University Press)

[10] Wick G-C, Wightman A S and Wigner E P 1952 Phys. Rev. 88101 Ballentine L E 1998 Quantum Mechanics: A Modern Development (Singapore: World Scientific)

[11] Weinberg S 1996 The Quantum Theory of Fields, vol 1 (Cambridge: Cambridge University Press).

[12] Haag R and Kastler D 1964 J. Math. Phys. 7848

[13] Werner R F 1989 Phys. Rev. A 404277

[14] Popescu S 1994 Phys. Rev. Lett. 72797

[15] Wootters W K 1998 Phys. Rev. Lett. 802245

[16] Sokolov V V, Brown B A and Zelevinsky V 1998 Phys. Rev. E 5856

[17] Thirring W 1958 Ann. Phys., NY 391 